\title{
Acoustic Resonance in a Model Ducted-Jet System
}

\author{
Victor D. Topalian* and Jonathan B. Freund $\ddagger$ \\ University of Illinois at Urbana-Champaign, Urbana, Illinois 61801
}

DOI: $10.2514 / 1.45191$

\begin{abstract}
Jet engines are tested in so-called test cells, in which the desired environmental conditions are controlled. The jet plume is exhausted out of the test cell, usually through a diffuser. Under certain testing conditions, high-intensity pressure fluctuations $(170 \mathrm{~dB})$ can arise in such facilities. Their frequency is close to that of an acoustic normal mode of the exhaust-diffuser, but the mechanisms driving the resonance are unclear. In the present work the resonance phenomenon is studied using numerical simulations of a model configuration, which shares the key features of actual facilities: an underexpanded supersonic jet, a finite-length solid-wall shroud surrounding the jet, and the receptivity of acoustic disturbances to excite jet instabilities at the nozzle. For this configuration a high-amplitude resonance, qualitatively similar to that of experiments in actual facilities, is observed for a symmetric overexpanded $M_{\text {jet }}=1.2$ jet. This strongly resonant case is contrasted with a nonresonant ducted $M_{\text {jet }}=1.5$ jet in the same geometry, as well as with a free jet at $M_{\text {jet }}=1.2$. The hydrodynamic mechanism of this resonance is studied using linear stability analysis. The presence of excited acoustic modes of the duct is revealed by the Fourier analysis of the data. A numerical experiment shows that slight artificial damping of just the most excited acoustic mode suppresses the resonance.
\end{abstract}

\section{Introduction}

$\mathbf{J}$ ETengines are tested in facilities like that shown schematically in Fig. 1. The engine is placed in a so-called test cell, in which the desired sea-level or altitude environmental conditions can be specified. The jet plume is exhausted out of the test cell, often through a diffuser. These gases are then carried downstream, cooled, and released to the atmosphere.

It has been reported in several studies [1-4], that under certain testing conditions high-intensity pressure fluctuations are observed in such facilities. The intensity of the fluctuations in the exhaust system reach, in some cases, nearly $170 \mathrm{~dB}$, which is beyond the acceptable limit for the installations, and so the tests are stopped to prevent structural damage. These events occur at specific testing conditions but their mechanism is not fully understood and the conditions leading to resonance can not currently be anticipated. The frequency of the fluctuations is in general close to that of an acoustic normal mode of the facility, typically that of the exhaust-diffuser, but the mechanism driving the resonance is uncertain.

There is to date no means to predict whether or not this highamplitude resonance will occur for a given set of testing conditions, or to anticipate the intensity under resonance [4]. So far, these resonances have been suppressed by trial-and-error procedures, either by changing the flow testing conditions (e.g., changing the amount of water spray injected to cool the diffuser walls or the amount of coflow) or the facility configuration (e.g., adding Helmholtz resonators to the diffuser, tuned to the frequency of the excited normal mode) [3]. Furthermore, it is not fully understood why this phenomenon occurs and what are the mechanisms driving it. From a practical point of view, such understanding should allow prediction of the regimes under which the resonance occurs and suggest effective mitigation procedures.

The aim of the present work is to study resonances of the type observed from a fundamental point of view. To this end, a simple,

Received 30 April 2009; accepted for publication 10 April 2010. Copyright (C) 2010 by the authors. Published by the American Institute of Aeronautics and Astronautics, Inc., with permission. Copies of this paper may be made for personal or internal use, on condition that the copier pay the $\$ 10.00$ per-copy fee to the Copyright Clearance Center, Inc., 222 Rosewood Drive, Danvers, MA 01923; include the code 0001-1452/10 and \$10.00 in correspondence with the CCC.

${ }^{*}$ Graduate Research Assistant, Department of Mechanical Science and Engineering; currently Postdoctoral Research Associate, Computational Science and Engineering.

${ }^{\dagger}$ Associate Professor, Department of Mechanical Science and Engineering, Department of Aerospace Engineering. two-dimensional ducted-jet model is designed to include the key features of the facilities needed for resonance so that we can simulate the flow numerically for different jet regimes. The use of numerical simulation allows us to visualize in more detail the flow variables as they go through a resonance cycle, allowing us to identify the features involved in the acoustic loop and to better characterize the acousticjet coupling. It is noted, however, that we expect to obtain a resonance cycle qualitatively similar to those in experiments, but we do not expect to match any quantitative result with this model configuration, since the details of the flow in this two-dimensional model are different.

The present study is structured as follows. In Sec. II, the model problem and the numerical method used are described. This model problem is a two-dimensional simplified version of the ducted-jet setup, which includes the shock-cell structure and shear layer of the imperfectly expanded jet, the acoustics of the duct, and the receptivity at the jet inflow. The numerical method that is used to solve the compressible-fluid flow equations is also outlined in this section. In Sec. III, the results of selected test cases are presented, and the main features of resonant and nonresonant regimes for this model problem are discussed. In Sec. IV, the data are analyzed in detail, and the implications of the results obtained in the model problem on the resonance phenomena in test-cell facilities are further discussed.

\section{Model Confined Jet}

\section{A. Model Configuration}

The model configuration is shown in Fig. 2. A jet of width $h$ with a coflow passes through a duct of width $H$ and length $L$.

The coflow spans the width of the duct and enters it through a reflective inflow. In this sense, the duct inflow resembles an ideal porous wall. Hence, the acoustic behavior of the duct is as if one end were closed and the other open (the inflow and outflow ends, respectively). Linear analytic models of these chambers have been designed using a similar assumption [2]. The jet and coflow exhausts to quiescent ambient conditions, with temperature $T_{\infty}$ and pressure $p_{\infty}$. The duct walls have thickness $w$.

The model problem includes the main features thought to play a role in the resonance phenomenon: an imperfectly expanded supersonic jet, its shock-cell structure, a shear layer separating the jet from the coflow, the acoustic confinement of the duct, and the receptivity at the inflow to acoustic perturbations. This allows the study of the problem at a fundamental level. However, it should also be clear that there are features of the full-scale facilities that are not captured by this model. The most important are expected to be the following: 


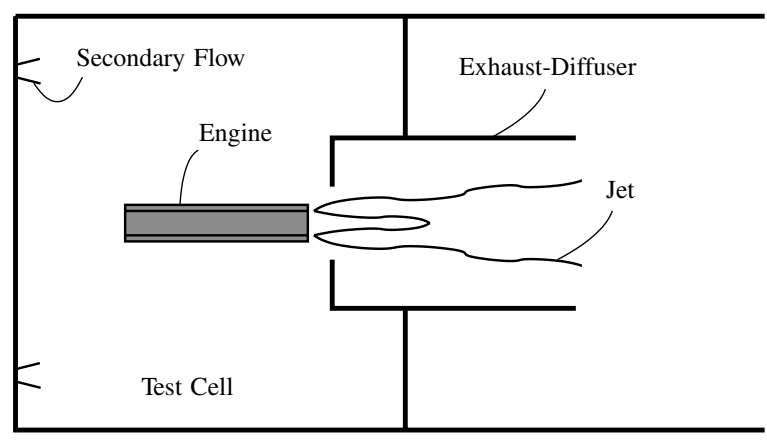

Fig. 1 Test-cell schematic.

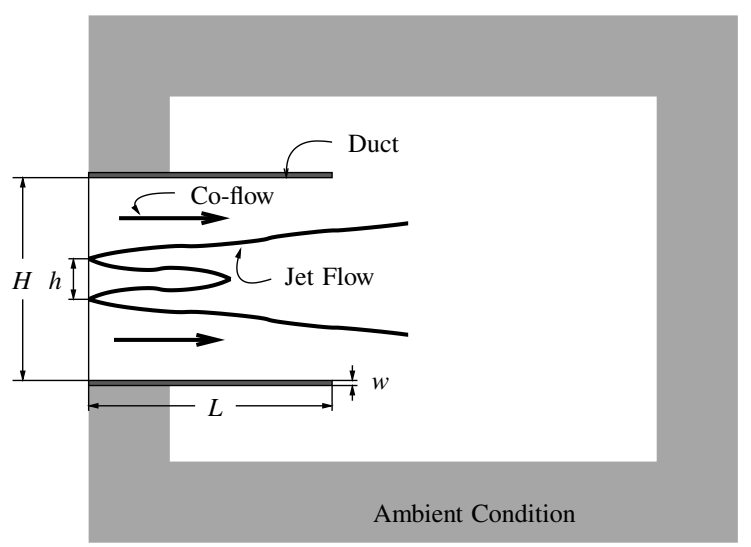

Fig. 2 Model problem schematic.

1) The flow is two-dimensional. In rectangular jet ejectors the flow is essentially two-dimensional, including the region where strong screech waves are generated [5]. Modes and features that are particular to cylindrical geometries will not be captured (for example, the helical or swirling movement of the jet). The effects of turbulence will also not be included. Turbulence produces broadband sound but is not thought to be an essential factor in the resonance.

2) There is no test cell represented upstream of the jet exhaust. Acoustics of the test cell might be a factor in actual facilities, but do not appear to be essential for it and therefore is not taken into account in this model configuration.

3) There is no nozzle. It has been suggested [3] that some resonant regimes are present due to strong oscillation in convergent-divergent nozzles, when the nozzle flow is transonic and a shock front is formed inside the nozzle. This specific mechanism is not taken into account by this model, but the inflow condition provides reflection of the acoustic perturbation into jet instabilities, which are expected to be important.

4) There is no engine, combustion, or species in the flow. Resonant regimes have been obtained in experiments without these elements. However, they might play a role in specific cases in actual test cells, which will not be assessed here.

In spite of these differences, the basic features believed to be involved in the phenomena are represented. Indeed, we shall see that our model geometry is sufficient to show strong resonances with characteristics matching those observed in full-scale facilities.

The flow is governed by the Navier-Stokes equations for compressible flows, which in nondimensional form are

$$
\begin{gathered}
\frac{\partial \rho}{\partial t}+\frac{\partial m_{i}}{\partial x_{i}}=0, \quad \frac{\partial m_{i}}{\partial t}+\frac{\partial u_{j} m_{i}}{\partial x_{j}}+\frac{\partial p}{\partial x_{i}}=\frac{1}{R e} \frac{\partial \tau_{i j}}{\partial x_{j}} \\
\frac{\partial E}{\partial t}+\frac{\partial u_{j} E}{\partial x_{j}}+p \frac{\partial u_{j}}{\partial x_{j}}=\frac{1}{R e} \tau_{i j} \frac{\partial u_{i}}{\partial x_{j}}-\frac{1}{(\gamma-1) \operatorname{RePr}} \frac{\partial q_{j}}{\partial x_{j}}
\end{gathered}
$$

where $\rho$ is the density, $u_{i}$ is the velocity, $p$ is the pressure, and $E$ is the internal energy, with $e=E / \rho$ as the mass specific internal energy. Invoking Stokes's hypothesis concerning the second viscosity coefficient, the viscous stress is $\tau_{i j}=\mu\left(u_{i, j}+u_{j, i}-\frac{2}{3} u_{k, k} \delta_{i j}\right)$. The mass flux is $m_{i}=\rho u_{i}$, the viscosity is $\mu=T^{0.76}$, and the diffusive heat flux is $q_{j}=-\kappa T_{, j}$, where $T$ is the temperature and $\kappa$ is the thermal conductivity. The thermodynamic relation $E=\rho T /(\gamma(\gamma-$ 1) $)=p / \gamma-1$ and state equation $p=\rho T / \gamma$ are as for a perfect gas. The nondimensionalization used is

$$
\begin{gathered}
\rho=\frac{\rho^{d}}{\rho_{\infty}} \quad x_{i}=\frac{x_{i}^{d}}{h} \quad t=\frac{t^{d} a_{\infty}}{h} \quad u_{i}=\frac{u_{i}^{d}}{a_{\infty}} \quad p=\frac{p^{d}}{\rho_{\infty} a_{\infty}^{2}} \\
T=\frac{T^{d}}{T_{\infty}} \quad e=\frac{e^{d}}{a_{\infty}^{2}} \quad \mu=\frac{\mu^{d}}{\mu_{\infty}} \quad \kappa=\frac{\kappa^{d}}{\kappa_{\infty}}
\end{gathered}
$$

where the superscript $d$ denotes dimensional variables. The $\infty$ subscript denotes an ambient condition. The nondimensional parameters are the Reynolds number $R e$, the Prandtl number $P r$, and the ratio of specific heats $\gamma$, given by

$$
\operatorname{Re}=\frac{\rho_{\infty} a_{\infty} h}{\mu_{\infty}} \quad \operatorname{Pr}=\frac{\mu_{\infty} c_{p}}{\kappa_{\infty}} \quad \gamma=\frac{c_{p}}{c_{v}}
$$

We take $\operatorname{Pr}=0.72$, and $\gamma=1.4$.

The inflow to the duct is modeled as an injecting no-slip wall with a jet profile, as shown in Fig. 3 .

The inflow profiles are all specified as

$$
B(y)=\left(B_{\text {jin }}-B_{\text {cof }}\right) \frac{g(y)-g(H / 2)}{g(0)-g(H / 2)}+B_{\text {cof }}
$$

with $B$ representing $u, T$, and $\rho ; B_{\text {in }}$ is the centerline value, $B_{\text {cof }}$ is the coflow value, and $g$ is

$$
g(y)=\tanh \left[\frac{2}{\delta_{w}}\left(y+\frac{h}{2}\right)\right]-\tanh \left[\frac{2}{\delta_{w}}\left(y-\frac{h}{2}\right)\right]
$$

where $\delta_{w}$ is a thickness parameter. In the limit of $\delta_{w} / h$ small, $H / h$ large, and constant density, the vorticity thickness for the shear layer of the jet is

$$
\delta_{w}=\frac{U_{\mathrm{jin}}-U_{\mathrm{cof}}}{\max _{y} U^{\prime}(y)} \approx\left|y_{0.5}-y_{0.96}\right|
$$

When $B=u$, the profile is also multiplied by

$$
\frac{1}{2}\left[\tanh \left[\frac{2}{\delta_{w 2}}\left(y+\frac{H}{2}\right)\right]-\tanh \left[\frac{2}{\delta_{w 2}}\left(y-\frac{H}{2}\right)\right]\right]
$$

to enforce a no-slip condition at the duct walls.

The inflow is fully determined by the expanded jet parameters $\left(M_{\text {jet }}=u_{\text {jet }} / a_{\text {jet }}\right.$ and $\left.p_{\text {jet }}, T_{\text {jet }}\right)$, the ratio between the mass flow rates

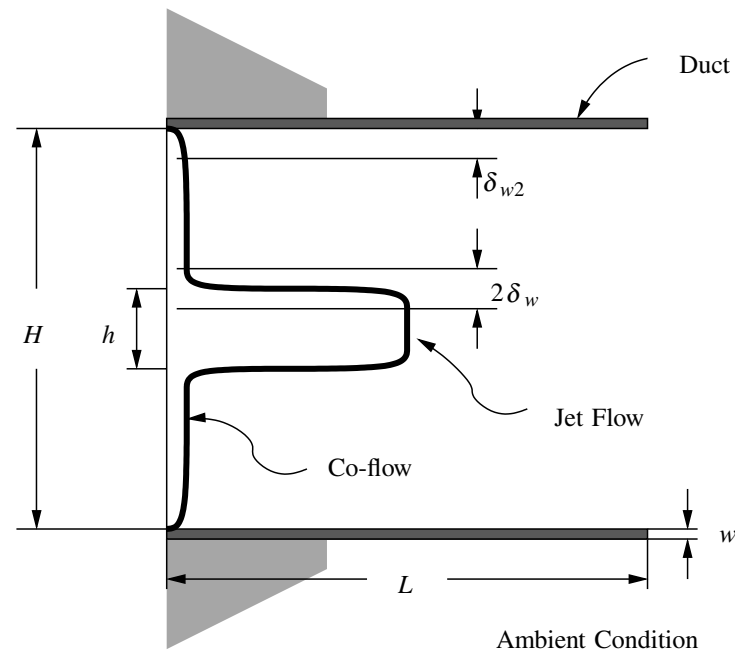

Fig. 3 Model configuration schematic: inflow details. 
of the coflow and the jet $\left(\beta=\dot{m}_{\text {cof }} / \dot{m}_{\text {jin }}\right)$, the coflow temperature $T_{\text {cof }}$, and the thickness parameters $\delta_{w}$ and $\delta_{w 2}$.

In the model, the jet flow is set as if it were exhausted from a nozzle, with an exit design Mach number $M_{e}=u_{\mathrm{jin}} / a_{\mathrm{jin}}$. Then the jet inflow condition is computed from the expanded jet parameters using isentropic flow relations. The mass flux for the jet at the inflow is $\left(m_{x}\right)_{\mathrm{jin}}=\rho_{\mathrm{jin}} u_{\mathrm{jin}}=\rho_{\mathrm{jin}} M_{e} \sqrt{T_{\mathrm{jin}}}$.

The mass flux of the coflow is obtained using the ratio between the flow rates of the jet and the coflow $\left(\beta=\dot{m}_{\text {cof }} / \dot{m}_{\text {jin }}\right)$. In actual facilities, as well as in experiments, this ratio usually ranges between 10 and 20\% [6]. Furthermore, it has been observed that the measured spectra in experiments is insensitive to this parameter. We parametrize the flow rates by considering uniform profiles in the jet region and in the coflow region, with widths 1 and $H-1$, respectively. Thus,

$$
\begin{gathered}
\rho_{\text {cof }} u_{\text {cof }}(H-1)=\beta \rho_{\mathrm{jin}} u_{\mathrm{jin}} \\
\left(m_{x}\right)_{\mathrm{cof}}=\beta \frac{1}{H-1}\left(m_{x}\right)_{\mathrm{jin}}
\end{gathered}
$$

The temperature of the coflow $T_{\text {cof }}$ is equal to the ambient temperature. Note that the inflow has both a supersonic and a subsonic region (corresponding to jet and coflow, respectively). The temperature profile at the inflow does not vary in time, while the density and pressure are steady in the supersonic region and dependent on time on the subsonic region. In this region they are obtained from the characteristic boundary condition imposed and the energy equation, respectively. The duct walls are no-slip and isothermal with temperature set equal to $T_{\infty}$.

\section{B. Numerical Method}

The model problem described in Sec. II poses several challenges from the numerical simulation point of view. In this flow, lowamplitude acoustic effects embedded in the higher amplitude hydrodynamic disturbances are important and have to be captured accurately in order for there to be a self-sustained resonant regime. Another important issue is the presence of large gradients within the flow due to the shock-cell structure in the jet core, which is the source of pressure waves that leak out of the jet (screech). Full details of the method are available elsewhere [7]. Here, we provide a basic outline of the scheme.

The equations are integrated in time using a standard fourth-order Runge-Kutta scheme, using a constant time step. In space, the equations are discretized on a staggered mesh and solved using finite differences. The staggered-mesh arrangement provides a robust formulation because of the discrete conservation of kinetic energy in the incompressible limit, in addition to mass and momentum [8]. It also provides improved resolution properties for finite difference derivative operators [9], although this benefit does not apply to every term in the flow. $\bar{A}$ formulation for compressible flows was developed by Nagarajan et al. [10], in which the superior stability properties over the regular and collocated mesh formulation were demonstrated. Boersma [11] implemented this discretization successfully for the case when chemical species are transported and reaction are coupled with the flow. In this formulation, only interpolation and first-derivative operators are needed. Highresolution (sixth-order) centered finite differences are used for interior points, where implicit schemes are used in the $y$ direction and explicit schemes are used in the $x$ direction. The explicit scheme facilitated the simpler one-dimensional decomposition of the domain for solution in parallel. The $x$ direction was chosen for parallel decomposition because more points are needed in the $x$ direction than in the $y$ direction due to a larger domain.

To simulate the shock-cell structure of the jet, we use the artificial nonlinear viscosity method developed by Cook and Cabot [12] and extended by Fiorina and Lele [13] to solve reacting flows. In this work the original formulation of Cook and Cabot [12] is used, since just a single nonreacting phase is included.

The incoming and wall boundary conditions inside the duct are enforced using a standard characteristic formulation [14]. The incoming and outgoing characteristics are schematically depicted in Fig. 4a. The inflow to the duct is modeled as a porous isothermal wall with constant mass flow rate. Hence, $m_{x}, m_{y}$, and $T$ are constant in time, with $m_{y}=0$. The inflow can be divided into a region of supersonic flow, which includes the jet core and part of the shear layers, and a region of subsonic flow, which includes the rest of the shear layers and the coflow. In the supersonic region there are no outgoing characteristics, hence all the primitive variables are determined from the steady inflow profile. In the subsonic region, following the procedure and nomenclature of Poinsot and Lele [14], the density is determined using

$$
\frac{\partial \rho}{\partial t}+d_{1}+\frac{\partial m_{y}}{\partial y}=0, \quad d_{1}=\frac{1}{a^{2}}\left[\mathcal{L}_{2}+\frac{1}{2}\left(\mathcal{L}_{5}+\mathcal{L}_{1}\right)\right]
$$

with

$$
\begin{gathered}
\mathcal{L}_{1}=(u-a)\left(\frac{\partial p}{\partial x}-\rho a \frac{\partial u}{\partial x}\right), \quad \mathcal{L}_{2}=u\left(a^{2} \frac{\partial \rho}{\partial x}-\frac{\partial p}{\partial x}\right) \\
\mathcal{L}_{5}=(u+a)\left(\frac{\partial \rho}{\partial x}+\rho a \frac{\partial u}{\partial x}\right)
\end{gathered}
$$

The amplitudes $\mathcal{L}_{2}$ and $\mathcal{L}_{5}$ are obtained from

$$
\frac{\partial T}{\partial t}+\frac{T}{\rho a^{2}}\left[-\mathcal{L}_{2}+\frac{1}{2}(\gamma-1)\left(\mathcal{L}_{5}+\mathcal{L}_{1}\right)\right]=0
$$

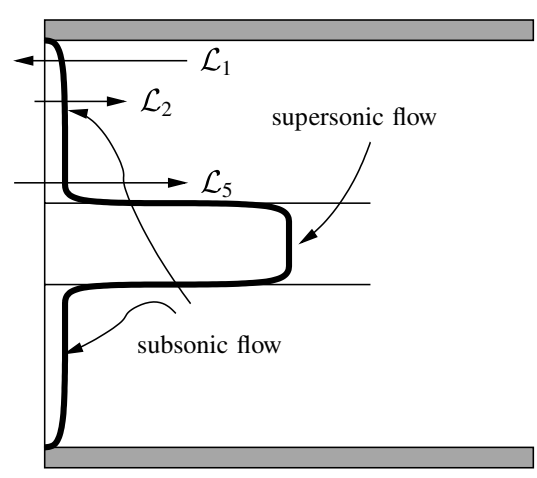

a)

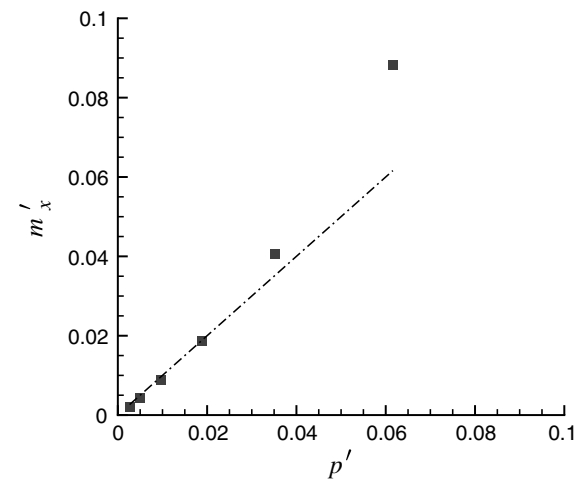

b)

Fig. 4 Details of jet inflow condition: a) incoming and outgoing characteristics for inflow within the duct and b) receptivity response of the inflow in the model problem. Maximum streamwise momentum fluctuation at $(0.5,0.5)$ versus maximum incoming pressure fluctuation at $(0.05,0.55)$. Each result corresponds to a different amplitude of the initial pressure pulse. 


$$
\frac{\partial m_{x}}{\partial t}+\frac{1}{a}\left[M \mathcal{L}_{2}+\frac{1}{2}\left[(M-1) \mathcal{L}_{1}+(M+1) \mathcal{L}_{5}\right]\right]=0
$$

where $M=u / a$ is the local Mach number. For constant $m_{x}$ and $T$, we obtain

$$
\mathcal{L}_{5}=\frac{1-M \gamma}{1+M \gamma} \mathcal{L}_{1}, \quad \mathcal{L}_{2}=\frac{\gamma-1}{2}\left(\mathcal{L}_{5}+\mathcal{L}_{1}\right)
$$

Since $\mathcal{L}_{1}$ is an outgoing wave, it can be computed from the flowfield directly. The internal energy is then computed using the equation of state after each RK4 substep. For the isothermal no-slip walls of the duct, the same approach is followed, as detailed by Poinsot and Lele [14].

The nonreflecting inflow and outflow conditions outside the duct are implemented using a stretched mesh buffer zone, in which the variables are low-pass filtered, and driven towards a target value by adding a forcing term of the form $\boldsymbol{F}=-\sigma(x, y)\left(\boldsymbol{q}-\boldsymbol{q}_{\text {target }}\right)$ added to the right-hand side of the Navier-Stokes equations, with $\boldsymbol{q} \equiv$ $\left\{\rho, m_{x}, m_{y}, E\right\}[\underline{15}]$.

The buffer zone is defined by the function $\sigma(x, y)=$ $\max \left\{\sigma_{x}(x), \sigma_{y}(y)\right\}$, with $\sigma_{x}(x)$ given by

$$
\sigma_{x}(x)= \begin{cases}\sigma_{\max }\left(\frac{x_{1}-x}{x_{1}-x_{\min }}\right)^{3} & x_{\min } \leq x \leq x_{1} \\ 0 & x_{1} \leq x \leq x_{2} \\ \sigma_{\max }\left(\frac{x-x_{2}}{x_{\max }-x_{2}}\right)^{3} & x_{2} \leq x \leq x_{\max }\end{cases}
$$

and with $\sigma_{y}(y)$ defined analogously. For the inflow region, seen in Fig. $4 \mathrm{a}, \sigma_{x}(x)=\sigma_{y}(y)=0$.

The explicit low-pass filter is defined as

$$
\hat{\hat{f}}_{i}=\frac{1}{4}\left(f_{i-1}+2 f_{i}+f_{i+1}\right)
$$

After each time step, the filter is applied in both the $x$ and $y$ directions, such that

$$
\begin{gathered}
\boldsymbol{q}^{\prime}=\left(1-\frac{\sigma_{x}(x)}{2 \sigma_{\max }}\right) \boldsymbol{q}+\left.\frac{\sigma_{x}(x)}{2 \sigma_{\max }} \hat{\hat{\boldsymbol{q}}}\right|_{x} \\
\boldsymbol{q}^{\prime \prime}=\left(1-\frac{\sigma_{y}(y)}{2 \sigma_{\max }}\right) \boldsymbol{q}^{\prime}+\left.\frac{\sigma_{y}(y)}{2 \sigma_{\max }} \hat{\boldsymbol{q}}\right|_{y}
\end{gathered}
$$

In this way, the fields are filtered gradually and primarily in the direction normal to the each inflow or outflow boundary. At the edge of the computational domain characteristic nonreflecting inflow and outflow conditions are applied [14].

Since receptivity at the inflow is an essential part of the resonance cycle, it is necessary to ensure that the inflow condition provides such receptivity of the shear layer to acoustic disturbances. Note that in the model configuration depicted in Fig. 3 the jet enters the domain right at the nozzle exit. In this sense, the inflow works as a virtual nozzle just outside the computational domain. To assess the viability of this approach, the receptivity at the inflow was tested and demonstrated as follows: a Gaussian pressure pulse was imposed in the freestream at $(3,1)$, in a free, symmetric, imperfectly expanded $M_{\text {jet }}=1.2$ jet. As expected, when the pressure disturbance reaches the inflow, the shear layer is perturbed, and a hydrodynamic wave is generated. This test was computed for several amplitudes of the initial pressure pulse. In Fig. $4 \mathrm{~b}$, the peak amplitude of the streamwise momentum fluctuation at $(0.5,0.5)$ is plotted against the peak pressure fluctuation of the incoming pulse at $(0.05,0.55)$. This shows the nearlinear response between the incoming pressure disturbance and the hydrodynamic wave generated by the inflow condition. The amplitude at $(0.05,0.55)$ is representative of the incoming pressure disturbance, outside the shear layer, whereas the amplitude at $(0.5$, 0.5 ) is representative of the hydrodynamic disturbance generated in the shear layer, in the linear region of growth. It is also noted that the tested pressure amplitudes at $(0.05,0.55)$ span the range of those in the simulation results presented in Sec. III. In view of these results, we conclude that the approach taken in the model problem is suitable for the purpose of the study of this model problem.

\section{Results}

\section{A. Symmetric Resonant Flow}

A set of test runs was performed in the model configuration, including jets in both symmetric and flapping jet regimes. Symmetry, when imposed, was enforced by a symmetry boundary condition at the jet centerline. From those test runs, high-amplitude pressure fluctuations (resonant) were observed for the underexpanded jet case at $M_{\text {jet }}=1.2$ in the symmetric regime (the complete set of parameters is presented in Sec. III.B). In the nonsymmetric cases, large flapping movement of the jet was obtained, and large hydrodynamic fluctuations were present in the region near the downstream end of the duct, as can be seen in Fig. 5, but no dominant tone was present in the frequency spectrum. In many cases, the jet became attached to one side of the duct, with a low-pressure recirculating region between the jet, the wall, and the inflow. The axisymmetric case is closer to the behavior observed in resonances in testing facilities, in the sense that dominant tones with frequencies similar to those of the normal modes of the duct were present, and hence we focus our study on this case.

For the numerical simulation of the symmetric flow only half of the spanwise domain is simulated, since $\rho, m_{x}$, and $E$ are even functions of $y$ about the centerline, $y=0$, and $m_{y}$ is an odd function of $y$. The

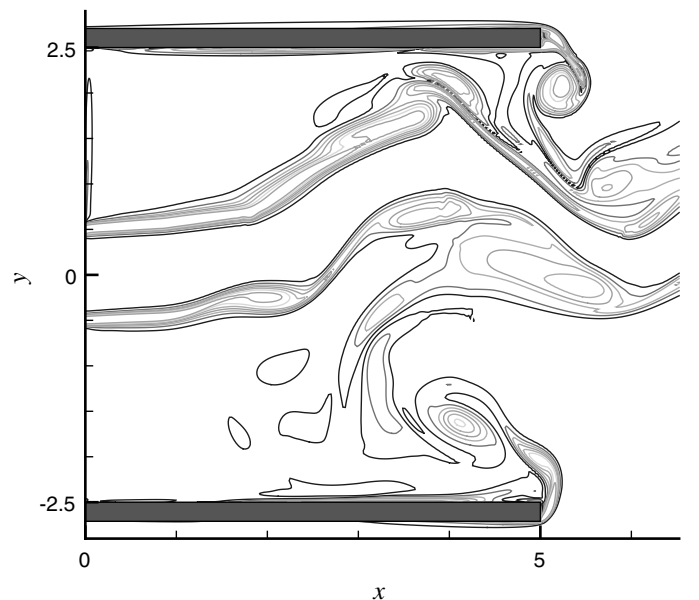

Fig. 5 Visualization of instantaneous vorticity magnitude for the asymmetric case. Contours: 6 levels from 0.5 to 6.5 .
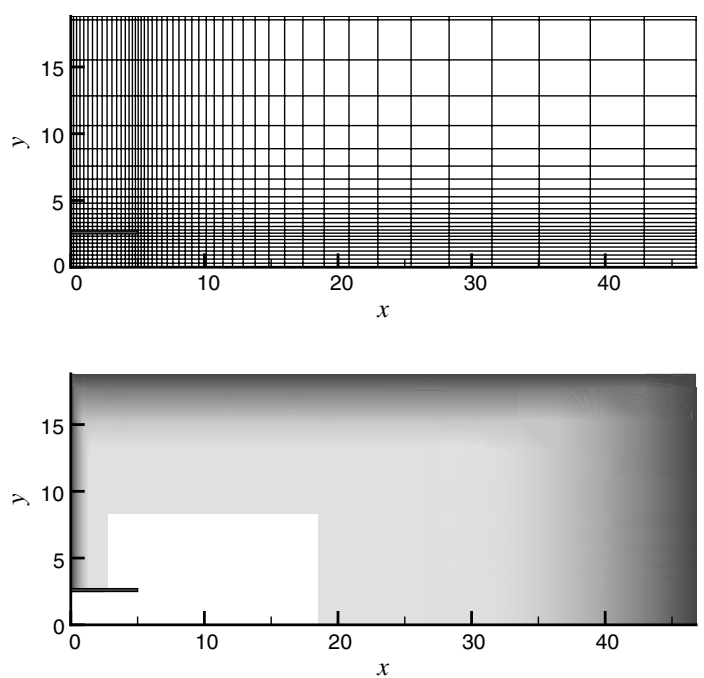

Fig. 6 Every 40th mesh point and grayscale indicating how $\sigma$ varies from 0 to 0.5 . 
Table 1 Intensity and frequency of the dominant tones obtained from the simulation of the model configuration

\begin{tabular}{cccc}
\hline \hline$M_{\text {jet }}$ & $M_{e}$ & Peak 1 & Peak 2 \\
\hline 1.1 & 1.25 & $150 \mathrm{~dB}, 0.20$ & 0.25 \\
1.2 & 1.35 & $159 \mathrm{~dB}, 0.21$ & 0.42 \\
1.3 & 1.44 & $150 \mathrm{~dB}, 0.21$ & 0.39 \\
1.4 & 1.54 & $153 \mathrm{~dB}, 0.21$ & 0.42 \\
1.5 & 1.63 & $146 \mathrm{~dB}, 0.21$ & 0.26 \\
1.6 & 1.73 & $125 \mathrm{~dB}, 0.21$ & 0.10 \\
\hline \hline
\end{tabular}

original interpolation, derivative and filtering operator were modified to automatically enforce these symmetries. In this way, the resolution properties of the implicit schemes are fully retained.

\section{B. Resonant Ducted Jet}

Simulations in the model configuration were performed in a grid of $670 \times 350$ points in the duct. The complete mesh has $1916 \times 1001$ points, where the domain including buffer zones spanned $0 \leq x \leq 46.8,0 \leq y \leq 18.8$. The mesh points were clustered near the wall and inflow boundaries, the duct exit, and the center of the duct. Figure $\underline{6}$ shows every 40th point in each direction of the nonuniform mesh, and a visualization of the $\sigma$ function that determines the buffer zone. The inner edges of the buffer zone are located at mesh points 337 and 1556 in the $x$ direction, corresponding to $x=2.5$ and 18.75 , respectively, and at point 855 in the $y$ direction, corresponding to $y=9.43$. Doubling the resolution for the $M_{\text {jet }}=$ 1.2 case did not change any of the conclusions regarding the resonance mechanisms.

The simulations were performed for $L / h=H / h=5$, $w / h=0.22, \quad T_{\text {jet }}=T_{w}=1, \quad \delta_{w}=\delta_{w 2}=1 / 12, \quad \beta=0.115$, $R e=1500$, and spanning a range of Mach numbers from 1.1 to 1.6. The jet Mach numbers and nozzle design Mach numbers are specified in Table $\underline{1}$, together with the peak frequencies observed in each case. Unless otherwise noted, the nondimensional frequency and the Strouhal number are

$$
f=\frac{f^{d} h}{a_{\infty}} ; \quad S t=\frac{f^{d} h}{U_{\text {jet }}}
$$

The results are given in terms of the nondimensional frequency since, as it is seen from Table 1, the dominant frequency is the same for all jet Mach numbers for the chosen parameters of the model problem. It will be shown later that this frequency, $f \approx 0.21$, is related to acoustic resonant modes of the duct.

In this section, the simulation of the $M_{\text {jet }}=1.2$ jet is referred to as the resonant case, because a high-amplitude resonance was observed in this case. The results of the $M_{\text {jet }}=1.5$ ducted jet (nonresonant) are also presented for comparison. Some results of the free (not ducted) $M_{\text {jet }}=1.2$ jet (the free jet) are also discussed.

The initial condition for the simulation was set to the inflow jet and coflow profile along the $x$ direction. The initial flow is transient, with the shock-cell structure forming and low-amplitude fluctuations appearing. In Fig. 7a the onset of resonance is observed, as the pressure at $(x, y)=\overline{(0.1,2.4)}$ increases in amplitude and becomes quasi-periodic. In Fig. 7b, the frequency components of pressure are also plotted, where $A_{H}=\sqrt{\hat{p} \hat{p}^{*}}$ is the absolute amplitude for each frequency.

It is observed in Figure $8 \mathrm{~b}$ that the amplitude of the dominant frequency is an order of magnitude higher in the resonant regime as compared with that of the free jet $(138 \mathrm{~dB}$ versus $159 \mathrm{~dB})$. It is also seen that the dominant frequency shifts from 0.340 in the free jet to 0.206 in the ducted jet.

In Fig. 9a the pressure history at $(0.1,2.4)$ is shown for the ducted $M_{\text {jet }}=1 . \overline{5}$ jet. It is seen in Fig. $9 \mathrm{~b}$ that the dominant frequency is approximately the same as in the $M_{\text {jet }}=1.2$ jet, but the intensity $(146 \mathrm{~dB})$ is much lower despite the higher jet speed. Furthermore, it is seen that the harmonic frequencies are excited in the resonant jet and
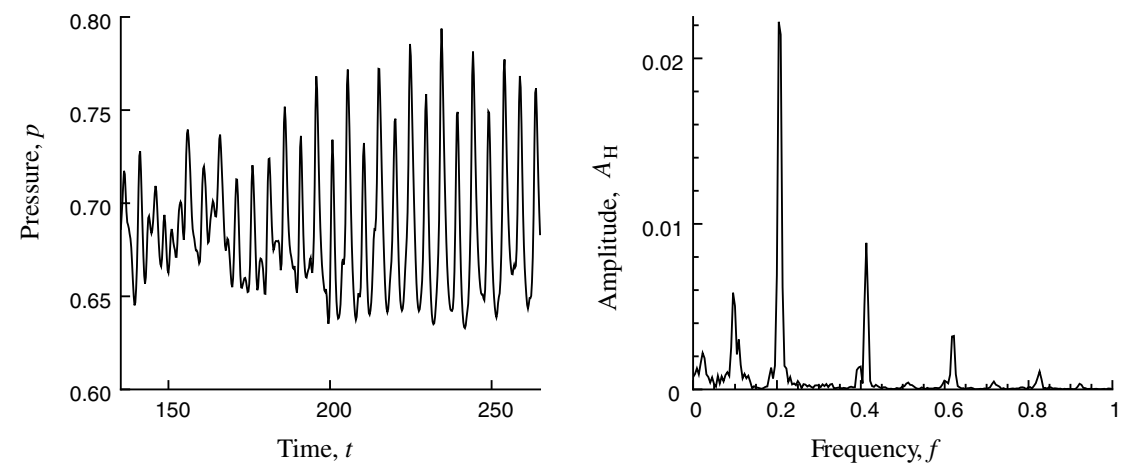

a)

b)

Fig. 7 Pressure at $(0.1,2.4)$ in the symmetric resonant regime: a) time trace and b) spectrum.
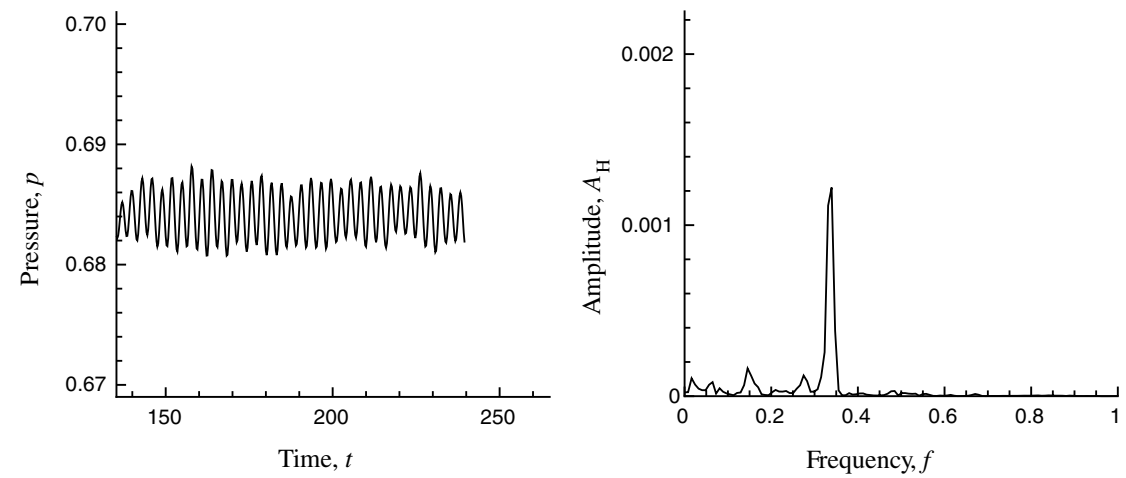

a)

b)

Fig. 8 Pressure at $(1.9,2.5)$ for the $M_{\text {jet }}=1.2$ free jet: a) time trace and b) spectrum. 


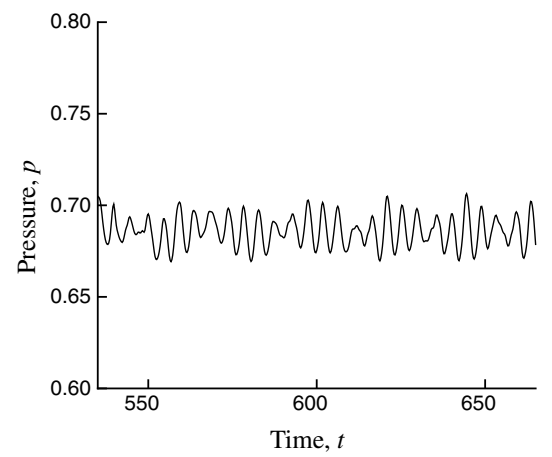

a)

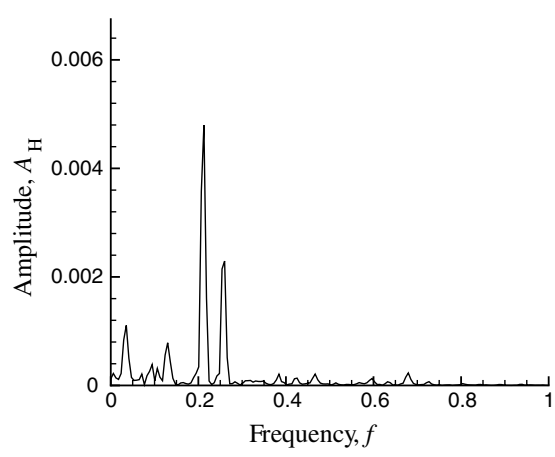

b)

Fig. 9 Pressure at $(0.1,2.4)$ in the nonresonant regime: a) time trace and b) spectrum.

that this does not happen in the $M_{\text {jet }}=1.5$ jet, where the secondary frequency in terms of amplitude is $f=0.26$. Note that in this case, unlike in the resonant jet, the harmonic frequencies of the dominant tone $(f=0.2)$ are not excited.

The resonance cycle is defined in terms of the dominant frequency present in Fig. 7b, $f=0.206$, and the images are labeled in terms of fractions of the period $T=4.85$. The flow is not exactly timeperiodic, but the features that will be highlighted are essentially the same for each cycle.

For comparison, mean quantities for the resonant, free, and nonresonant jets are shown in Figs. 10-12, respectively. The shockcell structure of the jets is clear. For the $\overline{M_{\text {jet }}}=1.2$ jet, the shocks are most clearly evident in Fig. 11. The end of the first, second, third, and fourth shock cells are located near $x=0.7,1.8,3.2$, and 4.5 . In the resonant case, shock cells are not observed beyond the third shock. In the nonresonant case, the shocks are more widely spaced and are located near $x=1.0,3.1$, and 5.2.

Contour lines of vorticity fluctuations are plotted over contour flood of rate of dilatation in Fig. 13, so the main vorticity structures and shock fronts in the flow can be seen. A main vortex (V1) can be seen in the $0.7 \mathrm{~T}$ image at about $x \approx 3$. This main vortex can be tracked backwards through the image series as the evolution of a small vorticity packet near the jet inflow $\left(\mathrm{V} 1^{\prime}\right)$ at about $x \approx 0.4$ in the same image. In addition to the main vortex (V1), a smaller vortical structure (V2) can be seen in the 0.9 T image, between $x=2$ and 3 . This structure can be seen to arise from the strip of vorticity between $x=0$ and 2 at about $0.5 \mathrm{~T}$. Between 0.5 and $0.9 \mathrm{~T}$ this strip merges into the small vortex V2. A shock front emanating from the jet can be
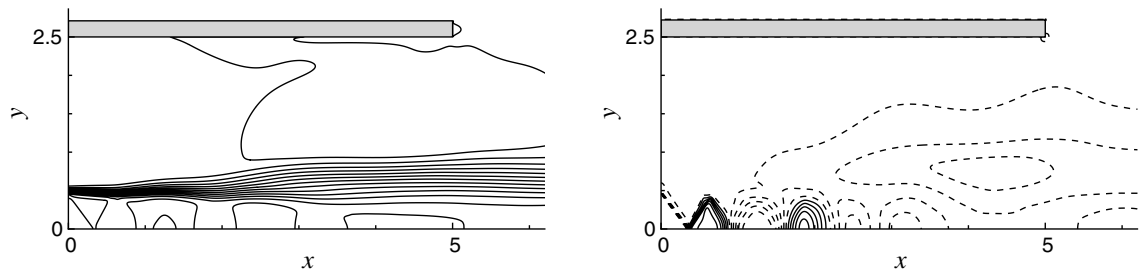

a) $\boldsymbol{u}_{\infty}$

b) $\boldsymbol{p}-\boldsymbol{p}_{\infty}$

Fig. 10 Contours of mean $u$ and $p-p_{\infty}$ for the $M_{\text {jet }}=1.2$ resonant jet. Contours: 7 levels from 0 to 1.2 for $u$ and 12 levels from -0.11 to 0.11 for $p-p_{\infty}$. Negative contours are dashed.

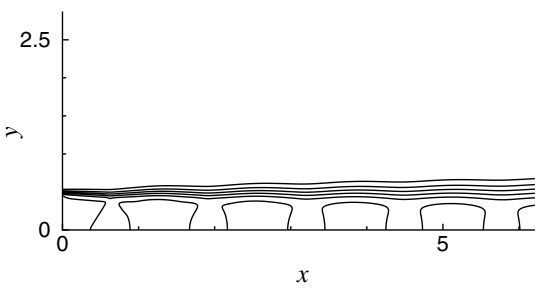

a) $u$

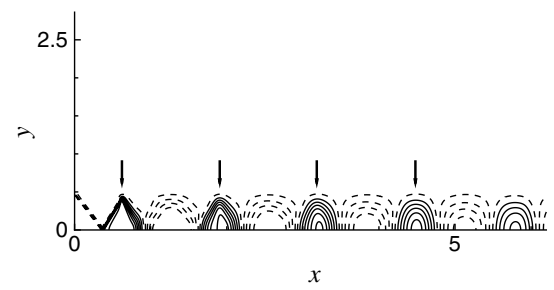

b) $p-p_{\infty}$

Fig. 11 Contours of mean $u$ and $p-p_{\infty}$ for the $M_{\text {jet }}=1.2$ free jet. Contours: 6 levels from 0.2 to 1.2 for $u$ and 12 levels from -0.11 to 0.11 for $p-p_{\infty}$. Negative contours are dashed.

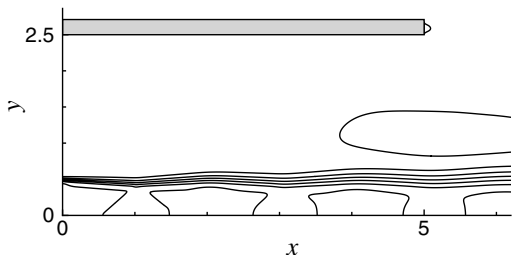

a) $\boldsymbol{u}$

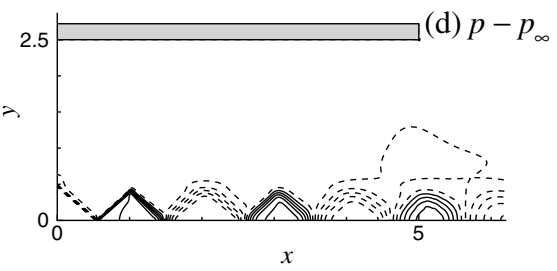

b) $p-p_{\infty}$

Fig. 12 Contours of mean $u$ and $p-p_{\infty}$ for the $M_{\text {jet }}=1.5$ jet. Contours: 7 levels from 0 to 1.5 for $u$ and 12 levels from -0.11 to 0.11 for $p-p_{\infty}$. Negative contours are dashed. 

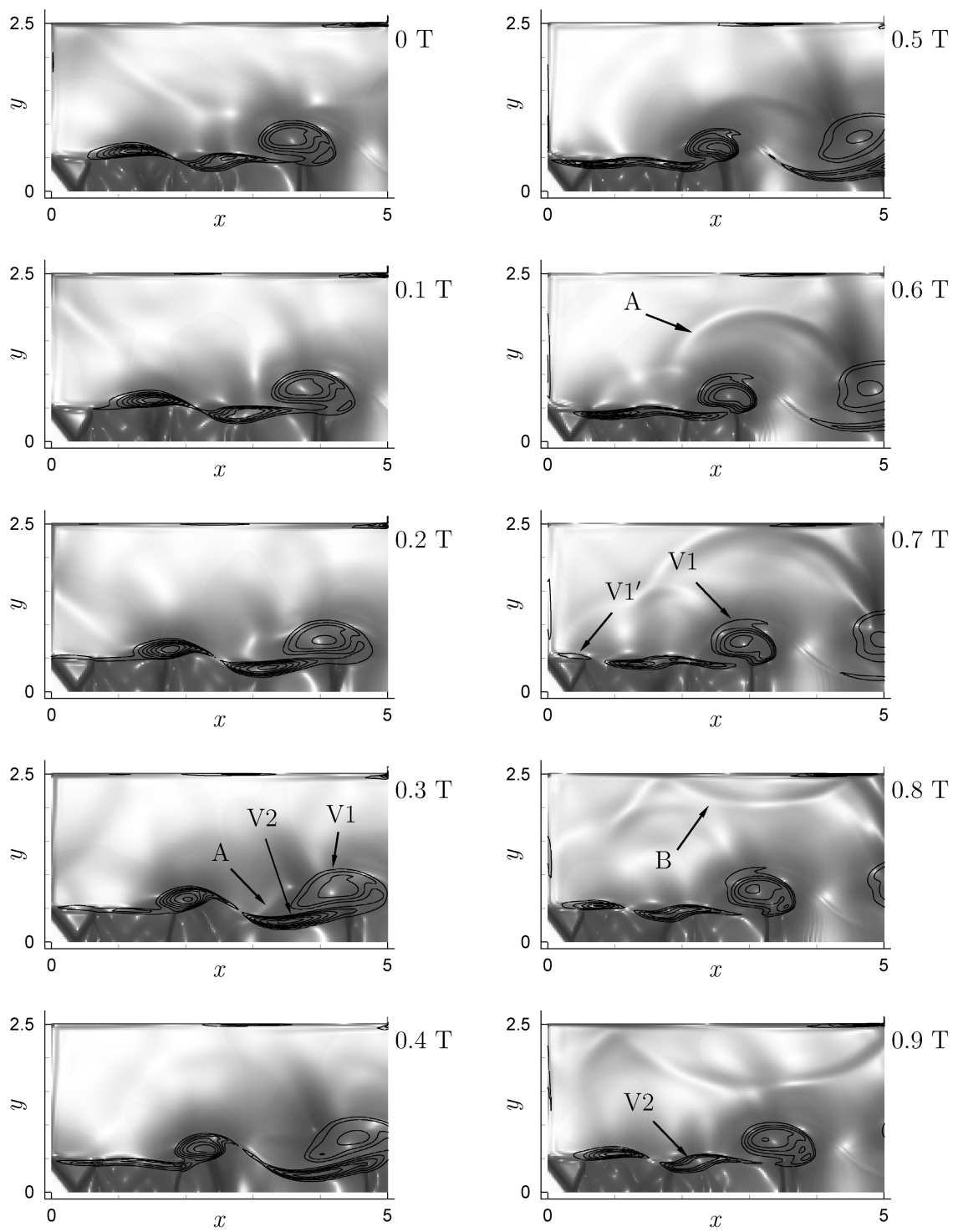

Fig. 13 Contour lines of vorticity fluctuations over contour flood of rate of dilatation. Contours: 13 levels from -1 to 0.2 for $\nabla u$ and 7 levels from 0.5 to 3.5 for $\omega^{\prime}$.

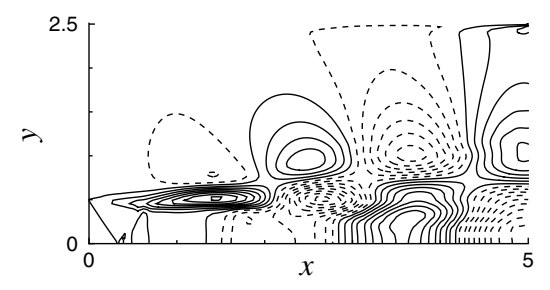

a) DNS at $f=0.2$

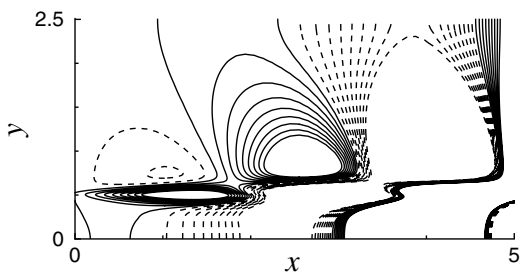

c) Linear stability at $f=0.2$

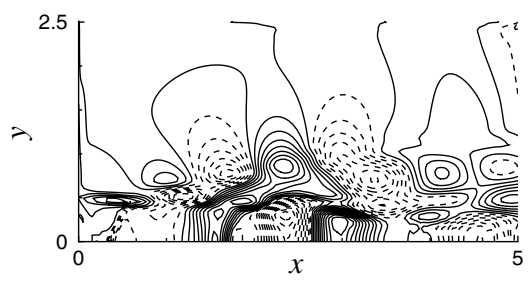

a) DNS at $f=0.4$

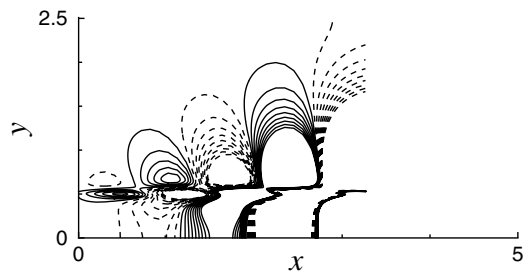

d) Linear stability at $f=0.4$

Fig. 14 Perturbation field for $u$ for the resonating ducted jet. Contours: (left) 18 levels from -0.07 to 0.07 and (right) 18 levels from -0.05 to 0.05 . Negative contours are dashed. 


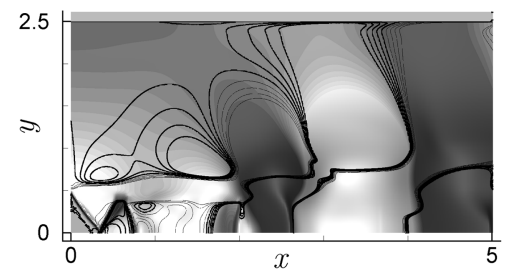

Fig. 15 Contours of streamwise velocity fluctuations over flood of pressure fluctuations at $0.5 \mathrm{~T}$.

seen in the 0.6 T image (labeled A in Fig. 13). This front can be tracked back to $0.3 \mathrm{~T}$, appearing to originate from the third shock cell, at $x \approx 3$. As it is shown in Fig. 13, this event correlates with the merging of V1 and V2. The weak shock front of $0.6 \mathrm{~T}$ can be tracked forward also, and it is seen to propagate in a circular wave and reflect in the wall of the duct, between 0.7 and $0.9 \mathrm{~T}$ (labeled B in Fig. 13). Part of the reflected wave propagates upstream and eventually reaches the inflow at about $0.3 \mathrm{~T}$. Visualizations of other quantities are provided elsewhere [7], and these show the same basic features.

\section{Discussion}

\section{A. Hydrodynamic Instability and Mechanisms of Resonance}

In this section, a local stability analysis considering a compressible inviscid flow in two dimensions is presented to show explicitly how the growth of hydrodynamic perturbations can be linked to instability modes of the jet. In doing this we assume parallel mean flow. A solution for each fluctuation variable is written as

$$
q_{1}(x, y, t)=\hat{q}(y) \exp [i(\alpha x+\omega t)]
$$

The mean velocity profile used for the streamwise velocity is as in Eq. (1), matching the inflow profile. To solve for spatial stability, we fix the real-valued frequency $\omega$ and seek for the values of $\alpha$ that satisfy the linear stability equations. The results of linear stability analysis are used to compute the perturbation field and compare the results with those of several direct numerical simulations (DNS) of the model configuration. The variable we compare is the fluctuation of the streamwise velocity, $u^{\prime}$. We solve for the stability problem at different $x$, using the local vorticity thickness $\delta_{w}$ computed from the DNS for each case. Then we estimate the perturbation field of the streamwise velocity from the stability analysis using

$$
u^{\prime}(x, y)=\hat{u}(y) \mid x \int_{0}^{x} \alpha \mathrm{d} x
$$

where we take the real part of $u^{\prime}$. The criterion to normalize $\hat{u}$ was $\{|\hat{u}|\}=\max \{\operatorname{Re}(\hat{u})\}=1$. This result is compared against the

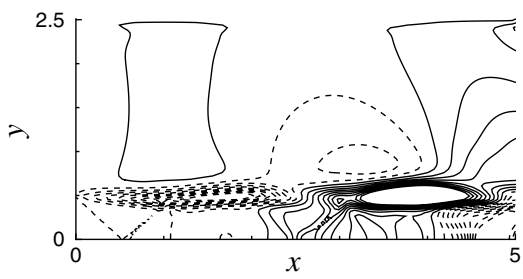

a) DNS at $f=0.20$

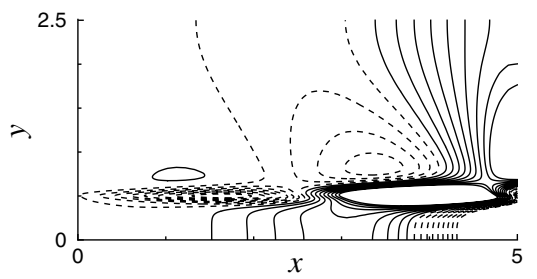

c) Linear stability at $f=0.20$ discrete Fourier transform (DFT) of the flowfield of the DNS at the same frequency. These transforms were performed using 200 flowfields separated in time by $\Delta t=50$. This covers approximately 10 and 20 periods of the frequencies $f=0.2$ and 0.4 , respectively.

The results for the resonating ducted jet at $M_{\text {jet }}=1.2$ are shown in Fig. 14 for the peak amplitude frequencies in Fig. $7 \mathrm{~b}$. In this case, a constant value of spreading rate for the vorticity thickness of $\mathrm{d} \delta_{w} / \mathrm{d} x=S=0.087$ was considered for all $x$.

Good agreement is seen between the DNS and from linear stability up to the streamwise position $x \approx 3$ for the $f=0.2$ mode and up to $x \approx 2$ for $f=0.4$. At this point, the perturbation pattern is different from that obtained from the linear stability analysis.

In Fig. 15, contour lines of velocity fluctuations are plotted over flood levels of pressure fluctuation from the DNS at $0.5 \mathrm{~T}$. We note the similarity in the velocity fluctuations field with Fig. 14d. From this similarity, it is inferred that both the fundamental and the harmonic frequencies, $f=0.2$ and 0.4 , are substantially present in the shear layer, and therefore, the perturbation at the inflow should occur at both of these frequencies. This is an important observation, since what we would expect is for the hydrodynamic fluctuations to be dominantly the product of perturbations at just the fundamental frequency. Furthermore, we note that these convective instabilities appear to evolve nonlinearly, since the similarity between the result from linear stability analysis and the DFT of the flowfield in Fig. 14 is lost at about $x=3$.

Referring now to Fig. 13, it follows that the vorticity fluctuations in the $0.5 \mathrm{~T}$ image are also a product of perturbations at both the fundamental and harmonic frequencies. Therefore, and considering the previous paragraph, we conclude that there is a nonlinear evolution of the vorticity fluctuations that, upon interacting with the shock-cell structure, produces the pressure pulse that appears to act as a periodic sound source in this resonance.

The results of the stability analysis for the ducted jet at $M_{\text {jet }}=1.5$ are shown in Fig. 16. This case also shows the presence of discrete frequencies. The higher peaks are in this case at $f=0.20$ and 0.26 , but the intensities are much lower than in the resonant case $\left(M_{\mathrm{jet}}=1.2\right)$. Note that since this jet does not resonate, the amplitude of perturbation at the inflow is much lower.

Note that in this case good agreement is obtained between the results from the DNS and the linear stability analysis along $x$ in all the domain of the duct. It is noted, however, that the amplitude of the perturbation (and then the amplification predicted) from linear stability is larger than from DNS. In this regard, it has to be noted that experiments $[16,17]$ show that the growth rate of shock containing jets is different to that of perfectly expanded jets. The consideration of these effects might give more accurate predictions regarding the growth rate of the hydrodynamic instabilities. Despite these differences, it can be concluded that the results from the local linear

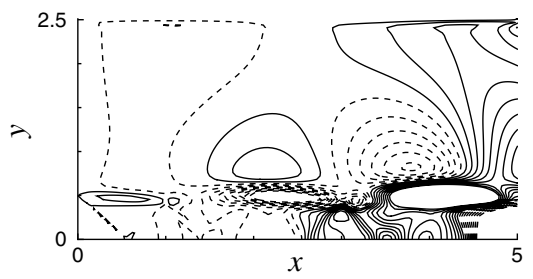

b) DNS at $f=0.26$

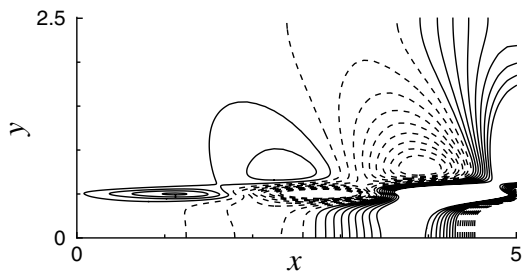

d) Linear stability at $f=0.26$

Fig. 16 Perturbation field for $u$ for the nonresonant $M_{\text {jet }}=1.5$ jet. Contours: (left) 18 levels from -0.025 to 0.025 and (right) 18 levels from -0.01 to 0.01. Negative contours are dashed. 
stability analysis provide a good representation of the hydrodynamic perturbation field observed in the simulation of the screeching symmetric nonresonant jet.

The integrated growth rate of the hydrodynamic instability wave is computed using the results of the linear stability analysis. The integrated growth rate, as considered by Tam et al. [18], is obtained from

$$
A=\exp \left[-\int_{0}^{x_{c}} \alpha_{i}(x) \mathrm{d} x\right]
$$

which is an integrated local growth rate of each mode $\alpha_{i}(x)$ between 0 and $x_{c}$, where $x_{c}$ is the location of the maximum wave amplitude. The result of this computation is shown in Fig. 17.

It has been suggested [18] that high-amplitude resonances are obtained when the most amplified hydrodynamic wave forms a resonant loop with a normal mode of the duct. It is seen in Fig. 17 that the growth rate for the $M_{\text {jet }}=1.2$ jet in the range $f=0.2$ to 0.4 is large compared with the growth rate at other frequencies. It is also noted, however, that the growth rates of the $M_{\text {jet }}=1.1$ case are even higher for the dominant tones $f=0.2$ and 0.26 , but the amplitudes from the simulation for this jet were about $10 \mathrm{~dB}$ lower than in the resonant case. Therefore, it is concluded that the criterion of the most amplified mode was not useful to anticipate a resonant regime for the present model configuration. Nevertheless, a high growth rate of the instability wave at the dominant frequency of the ducted jet seems to be a necessary condition for the resonance.

\section{B. Acoustic Mechanisms of Resonance}

1. Acoustics of the Duct and Resonant Frequencies

The equations of linear acoustics for quiescent homogeneous flow lead to the wave equation for pressure,

$$
\partial_{t} p_{m n}^{\prime}-a_{0}^{2} \nabla^{2} p_{m n}^{\prime}=0
$$

with general solution

$$
\begin{gathered}
p_{m n}^{\prime}=\operatorname{Re}\left\{\hat{p}_{m n} \exp \left(i\left(-\omega_{m n} t+\phi_{m n}\right)\right)\right\} \\
\hat{p}_{m n}=A_{m n} \exp \left(i\left(\alpha_{m} x+\beta_{n} y\right)\right)
\end{gathered}
$$

The complex amplitude $\hat{p}_{m n}$ depends on $x$ and $y$ with $\left(\alpha_{m}, \beta_{n}\right)$ the wave numbers in $x$ and $y$. The angular frequency is related to the spatial wave numbers by the dispersion relation,

$$
\omega_{m n}=2 \pi f_{m n}=a_{0} \sqrt{\alpha_{m}^{2}+\beta_{n}^{2}}
$$

For the duct in the model, normal mode solutions are obtained by applying the boundary conditions. The appropriate boundary conditions for our configuration are

$$
\left.\partial_{x} \hat{p}\right|_{(x=0)}=\left.\partial_{y} \hat{p}\right|_{(y=-H / 2)}=\left.\partial_{y} \hat{p}\right|_{(y=H / 2)}=0 \text { and }\left.\hat{p}\right|_{(x=L)}=0
$$

so it follows that

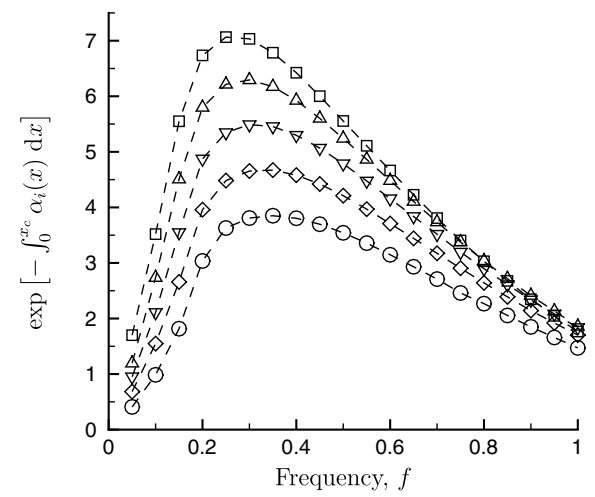

Fig. 17 Integrated growth rate for the simulated ducted jets: $M_{\text {jet }}=\square$ 1.1, $\triangle 1.2, \nabla 1.3, \diamond 1.4, \bigcirc 1.5$.
Table 2 Frequencies for the resonant case $\left(M_{\mathrm{jet}}=1.2\right)$ and acoustic normal modes

\begin{tabular}{lcl}
\hline \hline$f$ & $A_{H}$ & $(m, n): f^{(m, n)}$ \\
\hline 0.204 & 0.022 & $(0,2): 0.206$ \\
0.204 & 0.022 & $(2,0): 0.250$ \\
0.413 & 0.009 & $(1,4): 0.427$ \\
0.413 & 0.009 & $(3,2): 0.403$ \\
\hline \hline
\end{tabular}

$$
\begin{gathered}
\alpha_{m}=\frac{(2 m+1) \pi}{2 L}, \quad m=0,1,2, \ldots \\
\beta_{n}=\frac{n \pi}{H}, \quad n=0,1,2, \ldots \\
\hat{p}_{m n}=A_{m n} \Psi_{p}^{m n}(x, y)=A_{m n} \cos \left(\alpha_{m} x\right) \cos \left(\beta_{n} y\right)
\end{gathered}
$$

where $\Psi_{p}^{m n}$ us the pressure eigenfunction of the normal mode $(m, n)$. The corresponding velocity eigenfunctions are obtained from Eqs. (26) and (27) as

$$
\begin{gathered}
\hat{u}_{m n}=\frac{i A_{m n} \alpha_{m}}{\rho_{0} \omega_{m n}} \sin \left(\alpha_{m} x\right) \cos \left(\beta_{n} y\right) \\
\hat{v}_{m n}=\frac{i A_{m n} \beta_{n}}{\rho_{0} \omega_{m n}} \cos \left(\alpha_{m} x\right) \sin \left(\beta_{n} y\right)
\end{gathered}
$$

For our model configuration, which has $L=H=5$, the frequencies of the low-wave-number harmonics of the symmetric modes apparent in the simulation data are given in Table 2. It is not possible, of course, to distinguish between acoustic and hydrodynamic perturbations, especially in the vortical flow regions. We therefore consider regions dominated by the acoustic regime as those with irrotational flow and relatively low pressure fluctuations. For the cycle presented in Sec. III.B, the region near the top left corner of the duct domain has these characteristics (Fig. 18). The frequencies measured here (Fig. 7) are indeed close to those of normal modes, as listed in Table 2 .

The presence of acoustic normal modes is further substantiated by the DFT of the pressure fluctuations field, obtained as outlined in Sec. IV.A. The resulting real and imaginary fields are shown in Fig. 19. The clearest features of interest are the pressure nodes in the acoustic (upstream) region of the duct. The acoustic normal mode ( 0 , 2 ) of the duct has a pressure node at $y= \pm H / 4$. Similarly, the normal mode $(2,0)$ has pressure nodes at $x=L / 5$ and $3 L / 5$, the normal mode $(1,4)$ has pressure nodes at $y= \pm H / 8$ and $\pm 3 H / 8$ and $x=L / 3$, and the normal mode $(3,2)$ has pressure nodes at $y=$ $\pm H / 3$ and $x=L / 7,3 L / 7$, and $5 L / 7$. Such pressure nodes can be observed at approximately those positions in Fig. 19a-19d, respectively, in the region of the flow expected to be primarily acoustic.

A comparison between the phase fields from the DNS and the acoustic modes is presented in Fig. 20. The phase from the DNS data is obtained by direct calculation from the real and imaginary parts of the results of the DFT data. At each frequency we evaluate

$$
\tan \left(\phi_{f}(x, y)\right)=\frac{\operatorname{Im}\left\{\hat{p}_{f}(x, y)\right\}}{\operatorname{Re}\left\{\hat{p}_{f}(x, y)\right\}}
$$

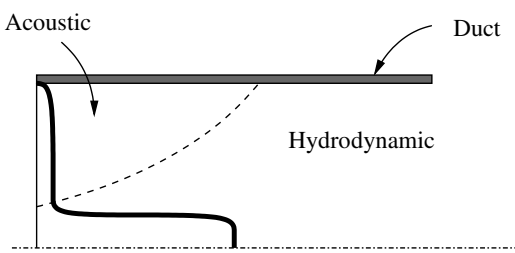

Fig. 18 Regions apparently dominated by acoustic and hydrodynamic fluctuations. 


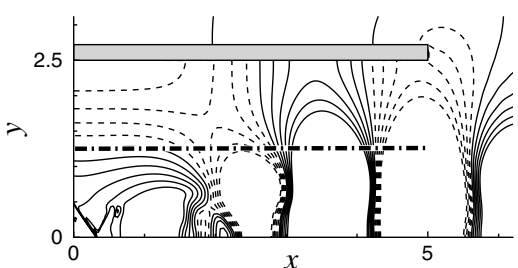

a) Real part, $f=0.2$

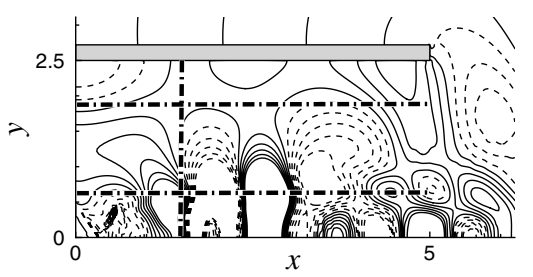

c) Real part, $f=0.4$

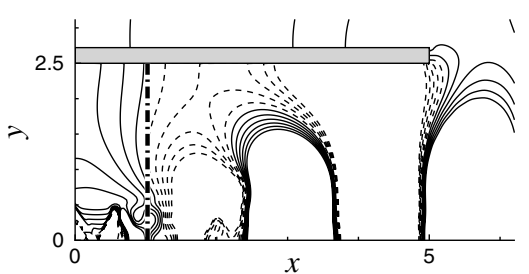

b) Imaginary part, $f=0.2$

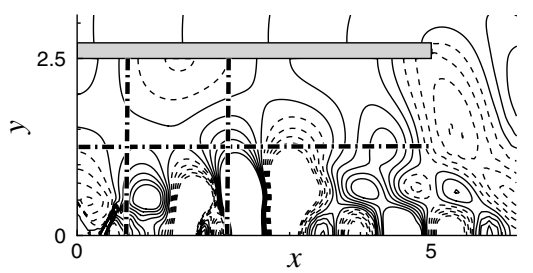

d) Imaginary part, $f=0.4$

Fig. 19 Contours of pressure fluctuations at discrete frequencies. Contours: (left) 11 levels from -0.02 to 0.02 and (right) 11 levels from -0.01 to 0.01 . Negative contours are dashed. The straight lines are the expected pressure node locations at these frequencies.

with $-\pi \leq \phi_{f} \leq \pi$. The computed phase fields are obtained assuming an acoustic field that is a superposition of two normal modes. In general, we can write

$$
\tan \left(\phi_{f}(x, y)\right)=\frac{\operatorname{Im}\left\{\sum_{i=1}^{N} \hat{p}_{m_{i} n_{i}} \exp \left(i\left(-\omega_{m_{i} n_{i}} t+\phi_{m_{i} n_{i}}\right)\right)\right\}}{\operatorname{Re}\left\{\sum_{i=1}^{N} \hat{p}_{m_{i} n_{i}} \exp \left(i\left(-\omega_{m_{i} n_{i}} t+\phi_{m_{i} n_{i}}\right)\right)\right\}}
$$

For the modes at $f=0.2$, the approximate absolute amplitudes for each mode were taken from the peak values in the acoustic region of the Fourier transformed results: $A_{0,2}=0.018$ and $A_{2,0}=0.005$. Also, a difference between the time phases $\left(\phi_{0,2}-\phi_{2,0}=\pi / 2\right)$ was imposed, since it follows from the transformed results that the modes are out of phase by about $\pi / 2$. The angular frequency of the normal modes was taken to be $f=0.2$. Similarly, for the modes at $f=0.4$, $A_{1,4}=0.006, A_{3,2}=0.003$, and $\left(\phi_{1,4}-\phi_{3,2}=\pi / 2\right)$.

The results plotted are taken at an arbitrary time $t$, chosen to match approximately the relative phases from the DNS data. The similarity between the patterns in the acoustic region of the duct is clear for both frequencies. In the downstream part of the duct the flow is dominated by hydrodynamic disturbances, and no matching is expected. It should be clear that this pattern for the phases can not be obtained from a single normal mode at either frequency, nor from a combination of normal modes at different frequencies. Therefore, this result is a strong indication that more than one mode is significantly excited at each frequency.

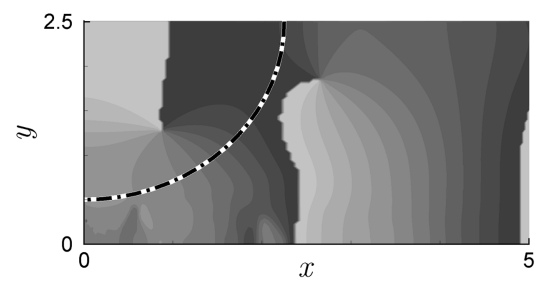

a) DNS, $f=0.2$

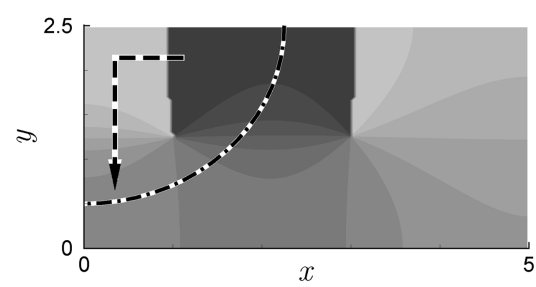

c) Normal modes, $f=0.2$

\section{Numerical Experiment: Suppression of Resonance}

A numerical experiment was carried out to understand the nature of the resonance by determining the amount of damping targeted to the acoustic modes needed to suppress it. A damping term was added to the Navier-Stokes equations to diminish the amplitude of the components of the second transverse mode of the duct $(0,2)$, the most energetic of the apparent modes in the acoustic field. We can anticipate that suppression of the acoustic mode should break the loop that leads to the strong resonance.

The damping term used for each variable is of the form $A_{i}^{(0,2)}(t) \Psi_{i}^{(0,2)}(x, y)$, with

$$
\begin{gathered}
\Psi_{\rho}^{(0,2)}(x, y)=\Psi_{E}^{(0,2)}(x, y)=\cos \left(\alpha_{0} x\right) \cos \left(\beta_{2} y\right) \\
\Psi_{m_{x}}^{(0,2)}(x, y)=\sin \left(\alpha_{0} x\right) \cos \left(\beta_{2} y\right) \\
\Psi_{m_{y}}^{(0,2)}(x, y)=\cos \left(\alpha_{0} x\right) \sin \left(\beta_{2} y\right)
\end{gathered}
$$

the normal mode eigenvectors. The time-dependent weight for the acoustic damping, $A_{i}^{(0,2)}(t)$, was obtained by considering a nonnormalized approximate projection of the fluctuations field of each variable into the normal mode at each substep of the time-marching scheme, as

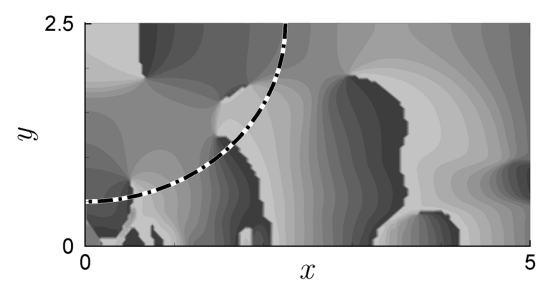

b) DNS, $f=0.4$

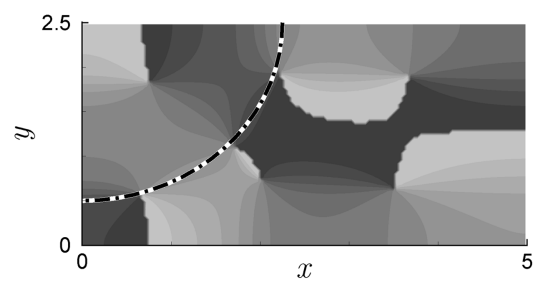

d) Normal modes, $f=0.4$

Fig. 20 Contours of relative phase for pressure fluctuations. Contours: 13 levels from $-\pi$ to $\pi$. 


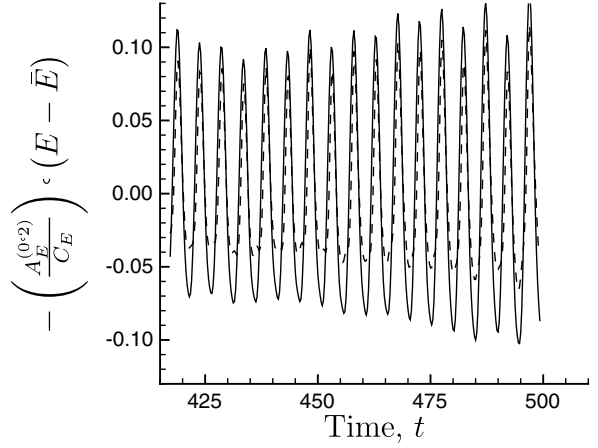

a)

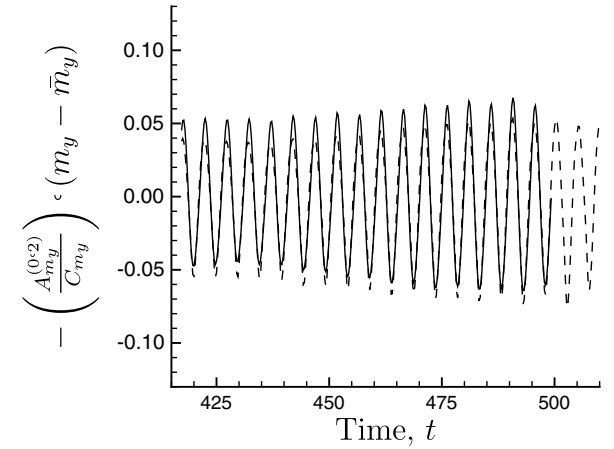

b)

Fig. 21 Fluctuations (dashed lines) and projections of fluctuations (solid lines) for a) $E$ and $b$ ) $m_{y}$. Fluctuations are considered at $(0.1,2.4)$ for $E$ and at $(0.1,1.25)$ for $m_{y}$.

$$
\begin{gathered}
A_{i}^{(0,2)}\left(t_{j}\right)=C_{i} \int_{x_{0}=0}^{x_{1}=1.25} \int_{y_{0}=1.25}^{y_{1}=2.5}\left(Q_{i}\left(x, y, t_{j}\right)\right. \\
\left.-\bar{Q}_{i}(x, y)\right) \Psi_{i}^{(0,2)}(x, y) \mathrm{d} y \mathrm{~d} x
\end{gathered}
$$

where $Q_{i}\left(x, y, t_{j}\right)$ is the instantaneous field, $\bar{Q}_{i}(x, y)$ is its mean field, and $C_{i}$ is a constant weight. We note that since it is not possible to separate the hydrodynamic field from the acoustic field, it is not possible to get an exact projection for each component of the acoustic field. Therefore, we use a nonnormalized approximate projection by computing the integral within the region $0 \leq x \leq 1.25$ and $1.25 \leq y \leq 2.5$. A sample of the result of the projection in Eq. (31) on fluctuations of internal energy and $y$ momentum is shown in Fig. 21, and compared with the actual fluctuations. The fluctuations are considered at $(0.1,2.4)$ for $E$ and at $(0.1,1.25)$ for $m_{y}$, close to the location of maximum amplitude for each variable for the acoustic normal mode.

The weight constants were set to $C_{\rho}=C_{E}=C_{m_{y}}=0.1$, and $C_{m_{x}}=0$. Regarding the choice for $C_{m_{x}}$, we expect the amplitude of the fluctuations in $m_{x}$ to be small compared with the other variables, since $\beta_{0}=\pi /(2 L)$ and $\alpha_{2}=2 \pi / H$, and therefore $u^{\prime 2} \ll v^{\prime 2}$. The results of the simulation with the damping terms included are shown in Fig. 22. It is seen that the amplitude of the fluctuations are greatly reduced after a few loop cycles. Furthermore, it is seen that the frequency of the highest-amplitude tone is also changed to near the first harmonic of the initial peak.

The change in total energy in the flow within the duct due to the damping terms can be computed directly via the instantaneous total energy in the duct, given by

$$
\mathcal{E}=\int_{y=0}^{y=H / 2} \int_{x=0}^{x=L}\left(\frac{1}{2 \rho}\left(m_{x}^{2}+m_{y}^{2}\right)+E\right) \mathrm{d} x \mathrm{~d} y
$$

As a first-order approximation, at any given time step, the total energy after applying the damping terms can be estimated as

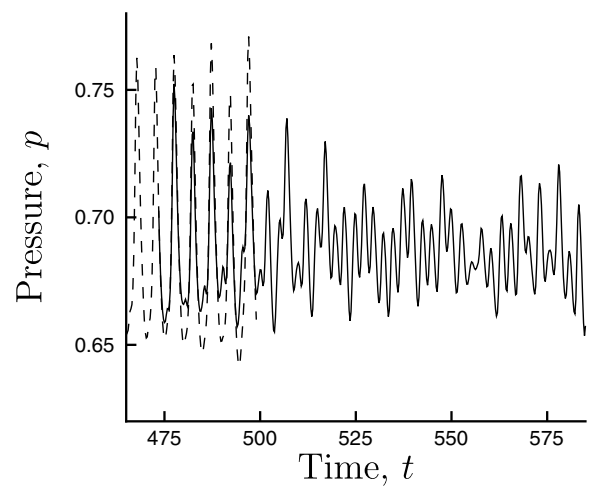

$$
\begin{aligned}
\mathcal{E} & +(\Delta \mathcal{E})_{\mathrm{damp}}=\int_{y=0}^{y=H / 2} \int_{x=0}^{x=L}\left[\frac { 1 } { 2 ( \rho + \Delta \rho ) } \left(\left(m_{x}+\Delta m_{x}\right)^{2}\right.\right. \\
& \left.\left.+\left(m_{y}+\Delta m_{y}\right)^{2}\right)+(E+\Delta E)\right] \mathrm{d} x \mathrm{~d} y
\end{aligned}
$$

with $\Delta q_{i} \approx A_{i}^{(0,2)}(t) \Psi_{i}^{(0,2)}(x, y) \Delta t$ the variation in the variable $q_{i}$ due to damping at the given time step. The variation in energy due to damping $(\Delta \mathcal{E})_{\text {damp }}$ can be compared with the variation of total energy in the duct $(\Delta \mathcal{E}=(\partial \mathcal{E} / \partial t) \Delta t)$ as a function of time. The result of this computation is shown in Fig. 23. It is seen that the amplitude of the variation in the energy due to damping is a small fraction (about $3 \%$ ) of the variation of the total energy in the duct due to the evolution of the hydrodynamic field. Therefore, it is concluded that the damping terms should not substantially directly affect the mean or hydrodynamic quantities of the resonant flow.

The mean flowfield after the damping is visualized in Fig. 24. It is seen that the features of the jet (e.g., shock-cell structure and mean values) appear unchanged when compared with Fig. 10, though the

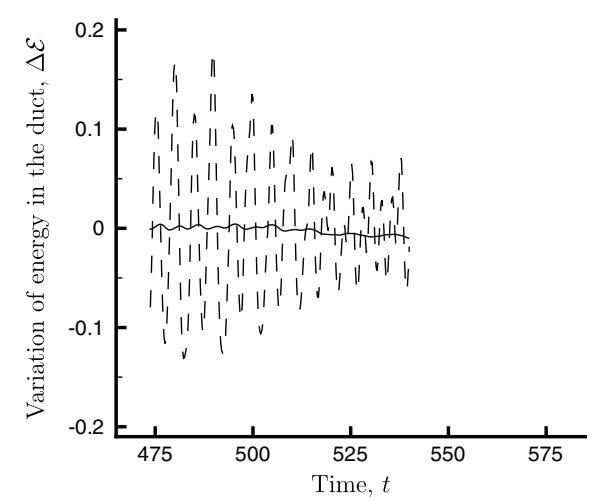

Fig. 23 Variation of total energy (dashed line) and energy dissipated due to damping (solid lines) in the duct as a function of time.

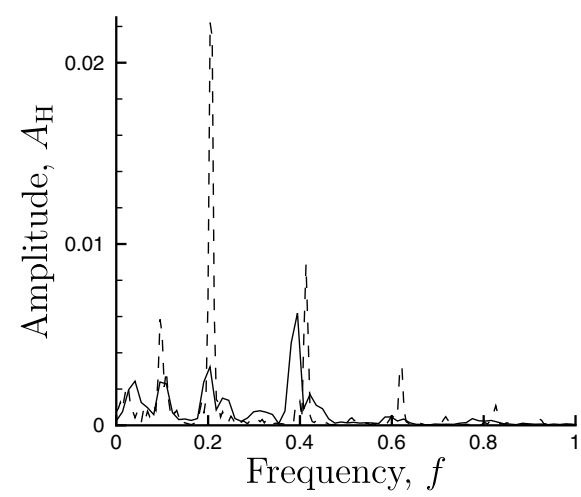

Fig. 22 Pressure at $(0.1,2.4)$ in the time and frequency domains for the resonant regime with (solid lines) and without (dashed line) acoustic damping. 


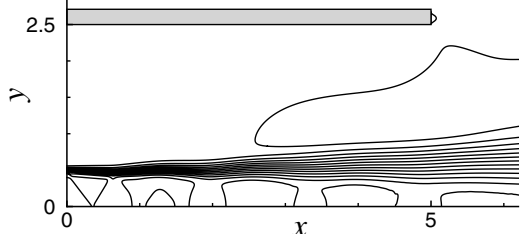

a) $u$

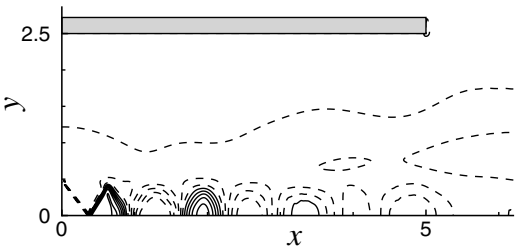

b) $\boldsymbol{p}-\boldsymbol{p}_{\infty}$

Fig. 24 Mean flow for the damped case: a) $x$ velocity $u$ (7 levels from 0 to 1.2), and b) $p-p_{\infty}$ (12 levels from -0.11 to 0.11 ). Negative contours are dashed.

shock-cell structure extends now beyond the third shock cell, as in the free jet, presumably because it is no longer disrupted by the strong oscillations of the hydrodynamic field.

\section{Resonance Cycle}

From the results obtained in this paper, the resonance cycle observed in the model configuration presented in this work can be described as follows. During a resonance cycle, the shear layer of the jet is perturbed at the inflow by pressure fluctuations related to the excitation of normal modes of the duct. The shear layer is excited at both the fundamental frequency (the frequency of highest amplitude, $f=0.2$ ) and its harmonic. The perturbations in the shear layer are convected downstream, evolve nonlinearly, and produce a pressure pulse, which acts as the noise source for this resonance. The pressure pulse is the product of the interaction of the hydrodynamic instability and the shock-cell structure of the jet. This acoustic pulse feeds energy back into the acoustic normal modes of the duct, which excite the shear layer at the inflow, closing the resonance cycle.

This description is similar to the explanations given by Tam et al. [18] and by Krothapalli and Hsia [19]. Tam et al. [18] proposed that a coupling occurs between the hydrodynamic instability and the acoustic modes of the duct and that the flapping motion of the jet was responsible for pumping energy back into the acoustic mode. The cycle is closed by the excitation of the shear layer near the nozzle by the acoustic modes. On the other hand, Krothapalli and Hsia [19] showed that in rectangular jet experiments, a main acoustic source can be identified at about the third shock cell. This source mechanism is activated when a vortical structure moves past the third shock cell. It was proposed that the pressure pulse emitted by the jet is reflected by the duct walls and that it perturbs the shear layer upon reaching the inflow. It was shown that acoustic modes of the duct were excited. They proposed that the acoustic modes act enhancing the mixing in the jet rather than producing perturbations at the inflow.

These different explanations consider sources of energy for the acoustic excitation of the duct and assign different roles to the excited modes. These issues need to be clarified further in order to develop a successful model to predict a resonant condition for a given set of parameters.

Regarding the effect of the variation of the duct dimensions, the resonant jet case was tested for $L=7.5$ and $H=5$ and for $L=5$ and

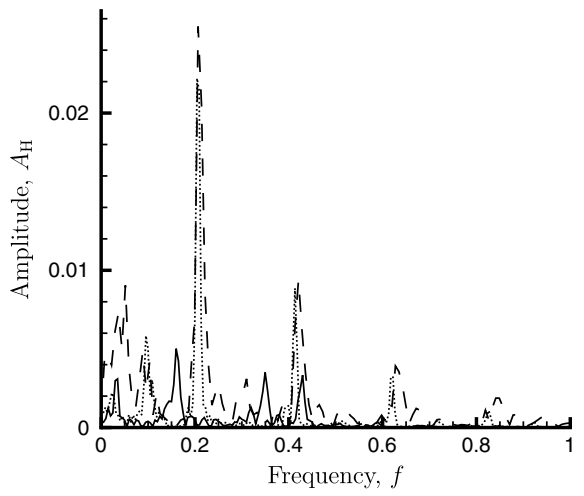

Fig. 25 Pressure in the frequency domain for a ducted $M_{\text {jet }}=1.2$ jet for $L=7.5$ and $H=5$ at $(0.1,2.4)$ (dotted lines), $L=5$ and $H=7$ at $(0.1$, 3.4) (solid lines), and $L=5$ and $H=5$ at $(0.1,2.4)$ (dashed line).
$H=7$. The results are plotted in Fig. 25. It is seen that the change in the length of the duct did not affect the resonant behavior, since the same frequencies are excited, and at approximately the same amplitudes. This fact is in agreement with the experimental results presented by Jones et al. [20], in which the same nondimensional frequencies were dominant for ducted jets, the ducts having the same diameter relative to the jet, but different lengths. On the other hand, the change in the width of the duct affected the resonant behavior, since both the frequencies and amplitudes observed are different.

It is noted, however, that the excited frequencies in the wider duct case ( $L=5$ and $H=7), f=0.16$ and 0.35 , are again close to those of the $(0,2)$ and $(1,4)$ transverse normal modes: $f_{(0,2)}=0.15$ and $f_{(1,4)}=0.32$. This strongly suggests that the dynamics of the jet in the duct is still dominated by the excitation of the transverse normal modes.

We note that, in spite of the large differences in shear-layer thickness and Reynolds number between the model problem and practical ducted jets, the fundamental and harmonic transverse frequencies of the duct are excited. This suggests that those parameters do not play an important role in the selection of the frequency at which the system will resonate, although they might be important to determine whether or not a resonance will occur. For example, in the model problem, it can be argued that a necessary condition to observe resonance is that the hydrodynamic modes of the jet must grow in space for both the fundamental and harmonic frequencies to allow the nonlinear interaction that produces the acoustic excitation to occur, where the growth rate depends on the shear-layer thickness. In this regard, resonant regimes might be present for ranges (as opposed to specific values) of shear-layer thicknesses and Reynolds number.

Last, we note that experimental results $[20,21]$ with similar basic configuration to the present model system showed that transverse eigenmodes are excited at intensities that can reach $170 \mathrm{~dB}$, with the fundamental mode being the most excited. While we clearly excite the acoustic duct modes in the same way as these experiments, the details are different here, as expected, because of the twodimensional symmetric flow.

\section{Conclusions}

In this work, flow-acoustic coupling in supersonic ducted jets was investigated using numerical simulations of a two-dimensional model configuration. The numerical method used solves the compressible Navier-Stokes equations, discretized on a staggered grid, using high-order finite differences. It also features shockcapturing capabilities necessary to resolve the shock-cell structure of the imperfectly expanded jet.

Several simulations were performed for fixed geometrical parameters of the duct and different jet Mach numbers. A resonant regime was identified for a symmetric $M_{\text {jet }}=1.2$ jet. It was shown that for this regime, the resonance cycle is composed of the following:

1) A pressure pulse emanates from the jet, which is the result of vortical structures interacting with the shock-cell arrangement of the jet.

2) Acoustic modes of the duct are excited by this noise source.

3) The perturbation of the shear layer of the jet at the inflow by these acoustic modes acts as feedback and closes the cycle to produce more hydrodynamic perturbations. 
The resonance cycle observed in the model configuration is similar to those proposed in previous studies $[18,19]$ with the difference that asymmetric jets and acoustic modes are dominant in actual setups. In this regard, the symmetry of the model setup affects the shear layer and acoustic modes of the duct excited, but not the phenomena or mechanisms present in the resonance cycle.

In the resonant regime, the hydrodynamic modes of the jet were identified. It was shown that the integrated growth rate of the hydrodynamic instability for the excited frequencies was relatively high, but that this criterion (matching of the excited frequency of the duct with the highest integrated growth rate of the hydrodynamic disturbance) did not necessarily predict a resonant regime for this model problem. The excited acoustic modes of the duct were identified as well, showing that both longitudinal and transverse modes were present in the acoustic field, both at the fundamental frequency of resonance and at its harmonic. Furthermore, it was shown using a numerical experiment that the resonance is suppressed when a damping term designed to remove a small amount of energy from the dominant acoustic mode is added to the equations. Also, for this model problem, the dominant frequency is strongly dependent on the duct width, rather than on its length, a result that is consistent with the importance of the excitation of the transverse modes of the duct, as it happens in actual facilities.

\section{Acknowledgments}

The support for this work from U.S. Air Force Office of Scientific Research, U.S. Department of Energy, and Center for Simulation of Advanced Rockets (CSAR) at the University of Illinois at UrbanaChampaign is gratefully acknowledged. The use of the Turing cluster maintained and operated by the Computational Science and Engineering Program at the University of Illinois is also gratefully acknowledged.

\section{References}

[1] Miller, V. R., "Reduction of the Acoustic Environment in an F100-PW100 Engine Test Cell," Proceedings of the National Conference on Noise Control Engineering, Noise Control Foundation, Poughkeepsie, NY, June 1981, pp. 257-260.

[2] Howe, M. S., "Self-Excited Oscillations in Jet Engine Test Cells," Journal of Fluids and Structures, Vol. 1, 1987, pp. 121-148. doi: $10.1016 / \mathrm{S} 0889-9746(87) 90230-1$

[3] Jones, R. R., III, and Lazalier, G. R., "The Acoustic Response of Altitude Test Facility Exhaust Systems to Axisymmetric and TwoDimensional Turbine Engine Exhaust Plume," 14th DGLR/AIAA Aeroacoustics Conference, Vol. 2, AIAA, Washington, D.C., May 1992, pp. 785-794.

[4] Sebourn, C. L., and Shope, F. L., "Research Summary on the AEDC ASTF C-2 Aeroacoustic Resonance Phenomenon," 11th AIAA/CEAS Aeroacoustics Conference (26th Aeroacoustics Conference), AIAA Paper 2005-2932, May 2005.

[5] Krothapalli, A., Hsia, Y. C., Baganoff, D., and Karamcheti, K., "The Role of Screech Tones in Mixing of an Underexpanded Rectangular Jet," Journal of Sound and Vibration, Vol. 106, No. 1, 1986, pp. 119143. doi:10.1016/S0022-460X(86)80177-8

[6] Ahuja, K. K., Jones, R. R., III, Tam, C. K., Massey, K. C., and Fleming, A. J., "Acoustic Interactions Between an Altitude Test Facility and Jet Engine Plumes-Theory and Experiments," Arnold Engineering
Development Center, TR-91-20, Arnold AFB, TN, 1992.

[7] Topalian, V., "Acoustic Resonances In Ducted Jet Systems," Ph.D. Thesis, Univ. of Illinois at Urbana-Champaign, Urbana, IL, 2009.

[8] Morinishi, Y., Lund, T. S., Vasilyev, O. V., and Moin, P., "Fully Conservative Higher Order Finite Difference Schemes for Incompressible Flow," Journal of Computational Physics, Vol. 143, No. 1, 1998, pp. $90-124$.

doi:10.1006/jcph.1998.5962

[9] Lele, S. K., "Compact Finite Difference Schemes With Spectral-Like Resolution," Journal of Computational Physics, Vol. 103, No. 1, 1992, pp. $16-42$. doi:10.1016/0021-9991(92)90324-R

[10] Nagarajan, S., Lele, S. K., and Ferziger, J. H., "A Robust High-Order Compact Method for Large Eddy Simulation," Journal of Computational Physics, Vol. 191, No. 2, 2003, pp. 392-419. doi:10.1016/S0021-9991(03)00322-X

[11] Boersma, B. J., "A Staggered Compact Finite Difference Formulation for the Compressible Navier-Stokes Equations," Journal of Computational Physics, Vol. 208, No. 2, 2005, pp. 675-690. doi:10.1016/j.jcp.2005.03.004

[12] Cook, A. W., and Cabot, W. H., "Hyperviscosity for Shock-Turbulence Interactions," Journal of Computational Physics, Vol. 203, No. 2, 2005 , pp. 379-385. doi:10.1016/j.jcp.2004.09.011

[13] Fiorina, B., and Lele, S. K., "An Artificial Nonlinear Diffusivity Method for Supersonic Reacting Flows with Shocks," Journal of Computational Physics, Vol. 222, No. 1, 2007, pp. 246-264. doi:10.1016/j.jcp.2006.07.020

[14] Poinsot, T., and Lele, S. K., "Boundary Conditions for Direct Simulation of Compressible Viscous Flows," Journal of Computational Physics, Vol. 101, No. 1, 1992, pp. 104-129. doi:10.1016/0021-9991(92)90046-2

[15] Freund, J. B., "Proposed Inflow/Outflow Boundary Condition for Direct Computation of Aerodynamic Sound," AIAA Journal, Vol. 35, No. 4, 1997, pp. 740-742. doi: $10.2514 / 2.167$

[16] Raman, G., and Rice, E. J., "Instability Modes Excited By Natural Screech Tones in a Supersonic Rectangular Jet," Physics of Fluids, Vol. 6, No. 12, 1994, pp. 3999-4008. doi: $10.1063 / 1.868389$

[17] Hu, T.-F., and McLaughlin, D. K., "Flow and Acoustic Properties of Low Reynolds Number Underexpanded Supersonic Jets," Journal of Sound and Vibration, Vol. 141, No. 3, 1990, pp. 485-505. doi:10.1016/0022-460X(90)90640-L

[18] Tam, C. K., Ahuja, K. K., and Jones, R. R., III, "Screech Tones From Free and Ducted Supersonic Jets," AIAA Journal, Vol. 32, No. 5, 1994, pp. 917-922. doi: $10.2514 / 3.12074$

[19] Krothapalli, A., and Hsia, Y. C., "Discrete Tones Generated by a Supersonic Jet Ejector," Journal of the Acoustical Society of America, Vol. 99, No. 2, 1996, pp. 777-784. doi:10.1121/1.414655

[20] Jones, R. R., III, Ahuja, K. K., Tam, C. K., and Abdelwahab, M., "Measured Acoustic Characteristics of Ducted Supersonic Jets at Different Model Scales," 31st AIAA Aerospace Sciences Meeting and Exhibit, AIAA Paper 1993-731, 1993.

[21] Massey, K. C., and Ahuja, K. K., "Flow/Acoustic Coupling in Ducted Jets," 19th AIAA Aeroacoustics Conference, AIAA Paper 1998-2211, Toulouse, France, June 1998. 